\title{
HYPERTROPHIC HASHIMOTO'S THYROIDITIS MIMICKING THYROID LYMPHOMA
}

\section{Niculescu ${ }^{1}$, Anda Dumitrascu $^{2}, \mathbf{R}$ lorgulescu $^{3}$, Catalina Poiana ${ }^{1}$}

${ }^{1}$ Department of Endocrinology, Carol Davila University, Bucharest, Romania;

${ }^{2}$ Department of Radiology, C. I. Parhon Institute of Endocrinology, Bucharest, Romania

${ }^{3}$ Department of Surgery, St. John Hospital, Bucharest, Romania

Correspondence at: d.niculescu@parhon.ro

\section{BACKGROUND}

$\checkmark$ Hashimoto's thyroiditis is a well known risk factor for primary thyroid lymphoma. The risk of PTL is between 40 and 80 times higher in patients with $\mathrm{HT}$;

$\checkmark$ The most common presentation of thyroid lymphoma is a rapidly enlarging, painless goiter. Other symptoms such as dyspnea, dysphagia, and hoarseness may arise as a result of the pressure effects of the mass. Cervical lymphadenopathy is present in the majority of cases.

\section{AIM}

$\checkmark$ To present a case of a rapidly enlarging goiter due to HT accompanied by enlarged lymph nodes and pressure on surrounding structures.

\section{CASE REPORT}

$\checkmark 85$ years old female;

$\checkmark$ Progressive enlargement of the thyroid since May 2015;

$\checkmark$ Dysphonia, dyspnoea;

$\checkmark$ Concomitant medication: indapamide, metoprolol and candesartan for high blood pressure
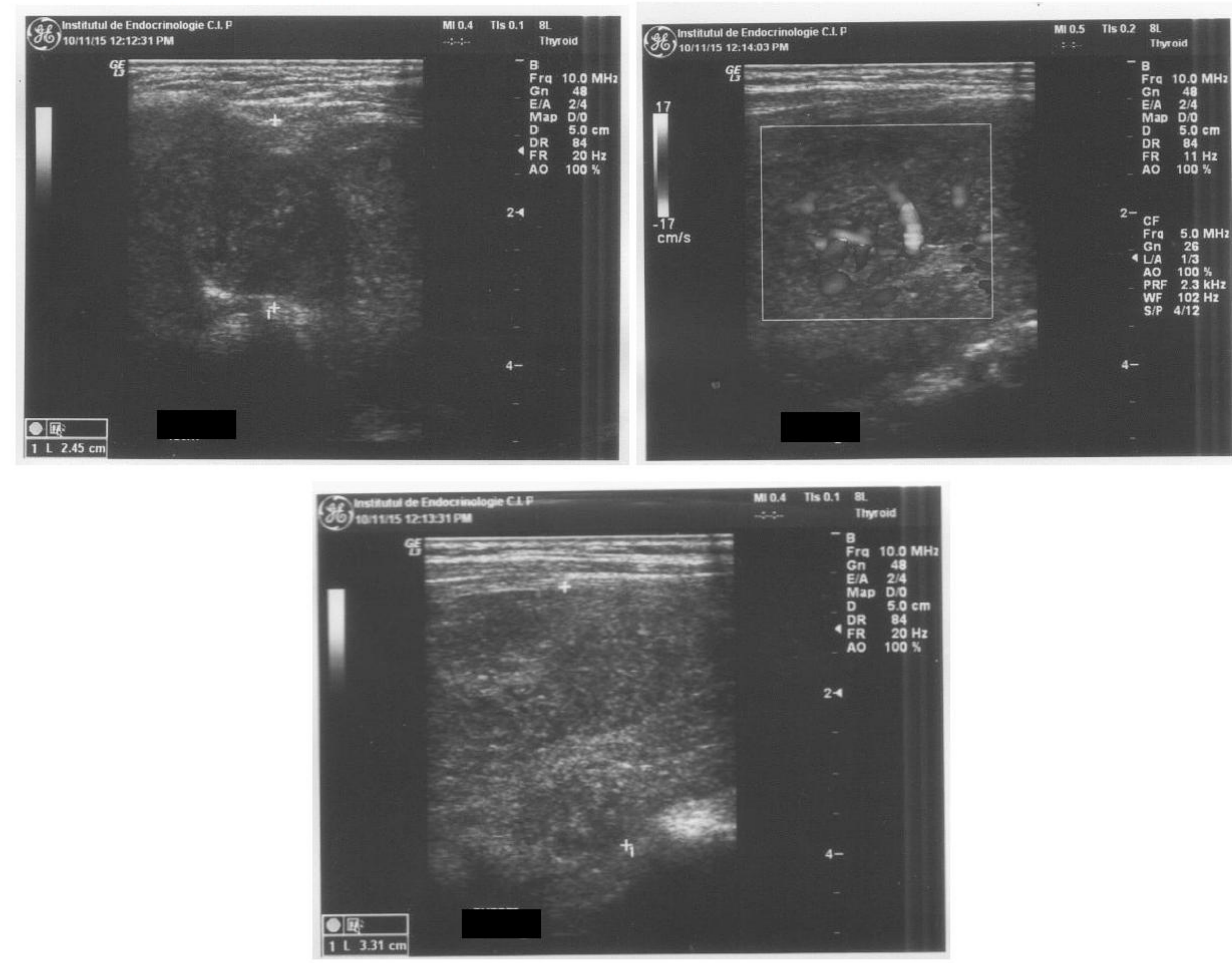

Thyroid ultrasound showing an intese hypoechoic, heterogeneous massive goiter. High blood flow on CFD examination; (upper left: istmus, upper right and lower: left lobe)

\begin{tabular}{lccc}
\hline \multicolumn{4}{l}{ Thyroid function and autoimmunity } \\
\hline & Jul 2015 & Nov 2015 & Jan 2016 \\
\hline TSH (mU/L) & 4.1 & 4.9 & 3.80 \\
\hline fT4 (pmol/L) & & 9.1 & 10.8 \\
\hline Anti-TPO (Ul/ml) & & $>1000$ & $>1000$ \\
\hline Anti-TGL (UI/L) & & $>3000$ & $>3000$ \\
\hline Calcitonin (pg/mL) & 2.2 & \\
\hline
\end{tabular}


Computed tomography showing a massive goiter (right lobe 88(v)/32(tr)/53(ap) mm; left lobe $91(\mathrm{v}) / 43(\operatorname{tr}) / 44(\mathrm{ap}) \mathrm{mm}$ ). Dense, homogeneous, iodofil structure.

\begin{tabular}{|c|c|c|}
\hline \multicolumn{3}{|c|}{ Pathology and immunohistocehmistry } \\
\hline & $\begin{array}{l}\text { Lymph node } \\
\text { (Aug 2015) }\end{array}$ & $\begin{array}{l}\text { Thyroid biopsy } \\
\text { (Dec 2015) }\end{array}$ \\
\hline Pathology & small B-cell NHL & chronic thyroiditis \\
\hline IHC & reactive lymph node & reactive lymphocytes \\
\hline \multicolumn{3}{|l|}{ Follicles } \\
\hline CD20 & + & + \\
\hline CD10 & + & \\
\hline CD23 & & + \\
\hline $\mathrm{BCL} 2$ & - & - \\
\hline Ki67 & High & + \\
\hline D1 cyclin & - & \\
\hline \multicolumn{3}{|c|}{ Parafollicular } \\
\hline CD3 & + & \\
\hline CD5 & + & + \\
\hline CD8 & & + \\
\hline
\end{tabular}

AS OF MAY 2016

$\checkmark$ Levothyroxine $50 \mu \mathrm{g} /$ day

$\checkmark$ No chemotherapy, no corticotherapy

$\checkmark$ No further growth of the goiter;

$\checkmark$ No further aggravation of the compression symtpoms;

$\checkmark$ No new symptoms.
CONCLUSIONS
Hypertrophic HT can clinically and imagistically mimic thyroid lymphoma;
An open biopsy of the thyroid may be required in these cases.

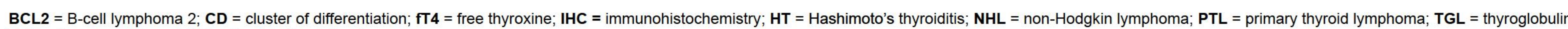
$\mathrm{TPO}=$ thyroperoxidase; $\mathbf{T S H}=$ thyroid stimulation hormone; 